\title{
Torsion of epiploic appendix following chiropractic movements: A case report
}

Seyed Ahmad Hashemi ${ }^{1}$, Rezvan Hosseinzadeh ${ }^{2}$, Morteza behnamfar ${ }^{3}$, and Mohammad Etezadpour $^{1}$

${ }^{1}$ Mashhad University of Medical Sciences

${ }^{2}$ Babol University of Medical Science

${ }^{3}$ North Khorasan University of Medical Sciences

February 7, 2022

\begin{abstract}
An epiploic appendix torsion is a rare event that can lead to acute abdominal pain and occurs at any age. Chiropractic is a type of complementary medicine performed explicitly by spinal manipulations. In this report, we presented a case who developed acute abdominal pain after spinal manipulation
\end{abstract}

\section{Hosted file}

Manuscript.docx available at https://authorea.com/users/377395/articles/555591-torsion-ofepiploic-appendix-following-chiropractic-movements-a-case-report

\section{Hosted file}

Figure 1.docx available at https://authorea.com/users/377395/articles/555591-torsion-ofepiploic-appendix-following-chiropractic-movements-a-case-report

\section{Hosted file}

Figure 2.docx available at https://authorea.com/users/377395/articles/555591-torsion-ofepiploic-appendix-following-chiropractic-movements-a-case-report 